\title{
Age-Related Pathophysiological Changes in Rats with Unilateral Renal Agenesis
}

\author{
Kohei AMAKASU ${ }^{1)}$, Katsushi SUZUKI ${ }^{1)}$, Kentaro KATAYAMA ${ }^{1)}$ and Hiroetsu SUZUKI ${ }^{1) *}$ \\ ${ }^{1)}$ Laboratory of Veterinary Physiology, Nippon Veterinary and Life Science University, 1-7-1 Kyonano-cho, Musashino, Tokyo 180-8602, \\ Japan
}

(Received 11 November 2010/Accepted 24 January 2011/Published online in J-STAGE 7 February 2011)

\begin{abstract}
Affected rats of the unilateral urogenital anomalies (UUA) strain show renal agenesis restricted to the left side. To determine whether unilateral renal agenesis is a risk factor for the progression of renal insufficiency, we studied age-related pathophysiological alterations in affected rats. Although body growth and food intake were normal, polydipsia and polyuria with low specific gravity were present at 10 weeks and deteriorated further with age. Blood hemoglobin concentrations were normal, though there was slight erythropenia with increased MCV and MCH. Although hypoalbuminemia, hypercholesterolemia, azotemia, and hypermagnesemia were manifested after age 20 weeks, neither hyperphosphatemia nor hypocalcemia was observed. Plasma Cre and UN concentrations gradually increased with age. Cre clearance was almost normal, whereas fractional UN excretion was consistently lower than normal. Proteinuria increased with age, and albumin was the major leakage protein. In addition to cortical lesions, dilated tubules, cast formation, and interstitial fibrosis were observed in the renal medulla of 50 week-old affected rats. Renal weight was increased 1.7-fold and glomerular number 1.2-fold compared with normal rats. These findings show that the remaining kidney in UUA rats is involved not only in compensatory reactions but experiences pathophysiological alterations associated with progressive renal insufficiency.

KEY WORDS: compensatory reaction, polyuria, proteinuria, renal agenesis, renal lesion.
\end{abstract}

There is evidence that one functioning kidney instead of two (i.e., a 50\% reduction in nephron mass) may not be sufficient to support normal renal function throughout life. For example, unilateral nephrectomy (UNx) in adult rats was found to reduce $\operatorname{GFR}[5,22]$, increase urinary volume [5], and decrease urinary maximum concentrating capacity [22]. Moreover, neonatal UNx resulted in systemic hypertension [34] and overt proteinuria [22], as well as a marked reduction in GFR. These observations suggest that the renal function of the remaining kidney deteriorates more severely when $\mathrm{UNx}$ is performed during the neonatal period than during adulthood. Unilateral renal agenesis (URA) is a common congenital anomaly of the kidney [24], occurring in $1: 1,000$ to $1: 500$ persons [33]. The incidences of proteinuria, hypertension, and renal insufficiency have been reported to be higher in human patients with URA and one normal kidney than in individuals with two normal kidneys [4]. The pathogenesis and course of these manifestations in the remaining kidney of patients with URA have not been well investigated, due, perhaps in part, to the absence of suitable animal models of human URA.

We previously reported the foundation and establishment of a new rat strain, the UUA (Unilateral Urogenital Anomalies) strain, characterized by URA and malformations of reproductive organs restricted to the left side. The URA was inherited as polygenic trait with the incidence of $20-60 \%$ in the strain [2]. The remaining right kidneys of affected rats were heavier than individual kidneys of normal rats. Interestingly, glomerulosclerosis, tubular dilation, and cast for-

\footnotetext{
* Correspondence to: Suzuki, H., Laboratory of Veterinary Physiology, Nippon Veterinary and Life Science University, 1-7-1 Kyonano-cho, Musashino, Tokyo 180-8602, Japan.

e-mail: hiroetsu@nvlu.ac.jp
}

mation were observed in the cortices of the remaining right kidneys of 30-week-old affected rats, suggesting the progression of renal dysfunction [2]. To evaluate the renal function of UUA rats and characterize them as an animal model of human URA, we examined age-related changes in their renal pathophysiological parameters, including renal excretion, urinary proteins, hematology, clinical chemistry, and renal histopathology.

Although UNx has been shown to induce compensatory renal growth $(\mathrm{CRG})$ of the remaining kidney $[5,15,21]$, it is unclear whether an increase in nephron number accompanies CRG [33]. Since increased nephron number has been reported in the remaining kidneys of patients with URA [13], we counted the number of glomeruli in the right kidneys of UUA rats to determine whether nephron hyperplasia contributes to CRG.

\section{MATERIALS AND METHODS}

Animals: All male rats used in this study were derived from the UUA strain [2]. Physiological data related to renal function were obtained from male rats aged $10,20,30,40$, and 50 weeks (in total, 31 normal and 34 affected). All male rats were categorized by palpation of the kidneys at 21 days of age, and the diagnosis was confirmed by necropsy [2]. The experimental procedures and care of animals were in accordance with the guidelines of the Animal Care and Use Committee of Nippon Veterinary and Life Science University $[29,30]$.

Measurements of food intake, water intake, and urinary volume: Male rats were kept individually in stainless steel metabolic cages (TOYO-RIKO Co., Ltd., Tokyo, Japan). Twenty-four hours food and water intake and $24 \mathrm{hr}$ urinary 
output were measured. Urine samples were centrifuged, and aliquots of the supernatants were stored at $-30^{\circ} \mathrm{C}[29,30]$. Urinary specific gravity (USG) was measured by a refractometer (SPR-N; ATAGO Co., Ltd., Tokyo, Japan).

Hematological and histological examinations: Blood samples were collected, and hematological examination was manually performed [29]. The plasma was removed and stored at $-30^{\circ} \mathrm{C}$ for clinical chemistry. Kidneys were removed and weighed. Right kidneys were processed for histological examination $[2,26,27,29,30]$. Praffin sections were stained with periodic acid Schiff (PAS) reaction [27].

Clinical chemistry: Plasma concentrations of albumin (ALB), total cholesterol (TCHO), calcium (Ca), inorganic phosphate (IP), and magnesium (Mg) were measured automatically (DRI-CHEM 3500V; Fujifilm Medical Co., Ltd., Tokyo, Japan) [28]. The plasma and urinary concentrations of creatinine (Cre) and urea nitrogen (UN) were measured using commercially available assay kit [26, 27, 29, 30].

Urinary protein concentration and electrophoresis: Urinary protein concentration was determined by the pyrogallol red method [23]. Five microliters of urine samples were fractionated by $12.5 \%$ SDS-polyacrylamide slab gel electrophoresis $[29,30]$. The gels were stained with Coomassie brilliant blue.

Determination of number of glomeruli in the right kidney: The numbers of glomeruli were counted in the right kidneys of 7 normal and 5 affected 10-11 week-old males. Glomeruli in the right kidney were counted by $\mathrm{HCl}$-maceration methods $[26,30]$. The total number of glomeruli in the right kidney of each rat was calculated.

Statistical analysis: Data are reported as mean and standard deviation (SD). Data at each time point were compared using unpaired Student's $t$-tests (Excel 2008 for Mac, Microsoft Corp, Redmond, WA, U.S.A.). Linear regression analyses were performed using Prism4 software (GraphPad Software, LaJolla, CA, U.S.A.) to determine age-related changes in renal function and to compare regression slopes and overall elevations [29].

\section{RESULTS}

Food and water intake and urinary volume (Table 1): Body weight gradually increased with age in both normal $\left(\mathrm{y}=5.607 \mathrm{x}+394.0, \mathrm{R}^{2}=0.507, P<0.0001\right)$ and affected $\left(\mathrm{y}=6.625 \mathrm{x}+347.1, \mathrm{R}^{2}=0.551, P<0.0001\right)$ rats, whereas food intake was almost constant over age in both normal (no significant correlation, $\mathrm{R}^{2}=0.042, P=0.276$ ) and affected $\left(\mathrm{R}^{2}=0.045, P=0.230\right)$ rats. There were no significant differences between normal and affected rats in body weight and food intake at all ages examined. Water intake $\left(\mathrm{y}=0.659 \mathrm{x}+31.66, \mathrm{R}^{2}=0.289, P=0.001\right)$ and urinary volume $\left(\mathrm{y}=0.772 \mathrm{x}+7.923, \mathrm{R}^{2}=0.440, P<0.0001\right)$ increased with age in affected rats but remained constant in normal rats (no significant correlation; $\mathrm{WI} \mathrm{R}^{2}=0.015, \mathrm{P}=0.522 ; \mathrm{UV} \mathrm{R}^{2}=0.017$, $P=0.498)$. Both water intake and urinary volume were significantly greater in affected than in normal rats at 10 (WI $P<0.05$; UV $P<0.001$ ), 30 (WI and UV $P<0.01$ ), 40 (WI $P<0.05$; UV $P<0.01$ ), and 50 (WI and UV $P<0.05$ ) weeks. USG decreased with age in affected $(y=-0.0005 x+1.051$, $\left.\mathrm{R}^{2}=0.305, P=0.0007\right)$ but not in normal $\left(\mathrm{R}^{2}=0.043\right.$, $P=0.2614)$ rats. USG was significantly $(P<0.01)$ lower in affected than in normal rats at 10,30, and 50 week-old, indicating a decrease in concentration capacity.

Renal weights (Fig. 1): The right kidneys of affected rats were significantly ( 1.7 to 2.4 fold) heavier than the right kidneys of normal at all ages. The remaining renal weight in affected rats was almost comparable to the total renal weight, of both kidneys, in normal rats. After age 30 weeks, the total renal mass was greater in affected than in normal rats, and the difference was statistically significant at 40 weeks of age $(P<0.05)$.

Hematological examination (Table 2): RBC (normal, $\mathrm{y}=0.032 \mathrm{x}+6.445, \mathrm{R}^{2}=0.333, P=0.0007$; affected, $\left.\mathrm{y}=0.044 \mathrm{x}+5.328, \mathrm{R}^{2}=0.575, P<0.0001\right)$ and Hct $\left(\mathrm{y}=0.084 \mathrm{x}+39.35, \mathrm{R}^{2}=0.135, P=0.042 ; \mathrm{y}=0.108 \mathrm{x}+36.13\right.$, $\mathrm{R}^{2}=0.185, P=0.013$ ) gradually increased with age in both normal and affected rats. However, their $\mathrm{Hb}$ concentrations were almost constant over time (no significant correlation; normal $\mathrm{R}^{2}=0.015, P=0.518$; affected $\mathrm{R}^{2}=0.014, P=0.515$ ).

Table 1. Age-related alterations in body weight, food and water intake, and urinary volume and specific gravity in normal and affected male rats

\begin{tabular}{ccccccc}
\hline Parameters & Phenotype & 10 weeks & 20 weeks & 30 weeks & 40 weeks & 50 weeks \\
\hline $\begin{array}{c}\text { Body weight } \\
(\mathrm{g})\end{array}$ & Normal & $384.1 \pm 25.1$ & $530.9 \pm 82.8$ & $626.8 \pm 29.0$ & $571.8 \pm 67.6$ & $667.2 \pm 62.4$ \\
& Affected & $359.9 \pm 39.3$ & $553.9 \pm 94.8$ & $582.7 \pm 64.1$ & $612.1 \pm 46.4$ & $604.5 \pm 38.6$ \\
\hline Food intake & Normal & $33.3 \pm 1.8$ & $29.3 \pm 4.3$ & $30.9 \pm 3.3$ & $28.6 \pm 2.2$ & $30.1 \pm 3.9$ \\
$(\mathrm{~g})$ & Affected & $32.4 \pm 3.1$ & $28.9 \pm 3.4$ & $31.0 \pm 2.7$ & $29.9 \pm 2.2$ & $30.2 \pm 2.5$ \\
\hline Water intake & Normal & $35.2 \pm 1.1$ & $35.0 \pm 4.8$ & $34.3 \pm 3.3$ & $34.7 \pm 4.8$ & $33.4 \pm 5.7$ \\
$(\mathrm{~g})$ & Affected & $\left.39.5 \pm 3.3^{\mathrm{a}}\right)$ & $39.0 \pm 7.4$ & $53.8 \pm 17.8^{\mathrm{b})}$ & $65.2 \pm 16.6^{\mathrm{a})}$ & $64.9 \pm 14.0^{\mathrm{a})}$ \\
\hline Urine volume & Normal & $11.7 \pm 0.6$ & $22.7 \pm 3.4$ & $15.1 \pm 2.2$ & $13.2 \pm 2.7$ & $15.6 \pm 4.1$ \\
$(\mathrm{~m} l)$ & Affected & $14.8 \pm 1.4^{\mathrm{c})}$ & $20.8 \pm 5.1$ & $31.2 \pm 15.8^{\mathrm{b})}$ & $38.3 \pm 3.6^{\mathrm{b})}$ & $43.8 \pm 1.5^{\mathrm{a})}$ \\
\hline USG & Normal & $1.057 \pm 0.008$ & $1.04 \pm 0.008$ & $1.052 \pm 0.006$ & $1.042 \pm 0.010$ & $1.051 \pm 0.009$ \\
& Affected & $\left.1.045 \pm 0.005^{\mathrm{e}}\right)$ & $1.044 \pm 0.010$ & $1.038 \pm 0.012^{\mathrm{e})}$ & $1.031 \pm 0.008$ & $1.027 \pm 0.007^{\mathrm{e})}$ \\
\hline
\end{tabular}

Each data is represented as mean \pm SD. Significantly higher [a) $P<0.05$, b) $P<0.01$, and c) $P<0.001$ ] and significantly lower [e) $P<0.01]$ than in aged matched normal rats by Student's $t$-tests. 


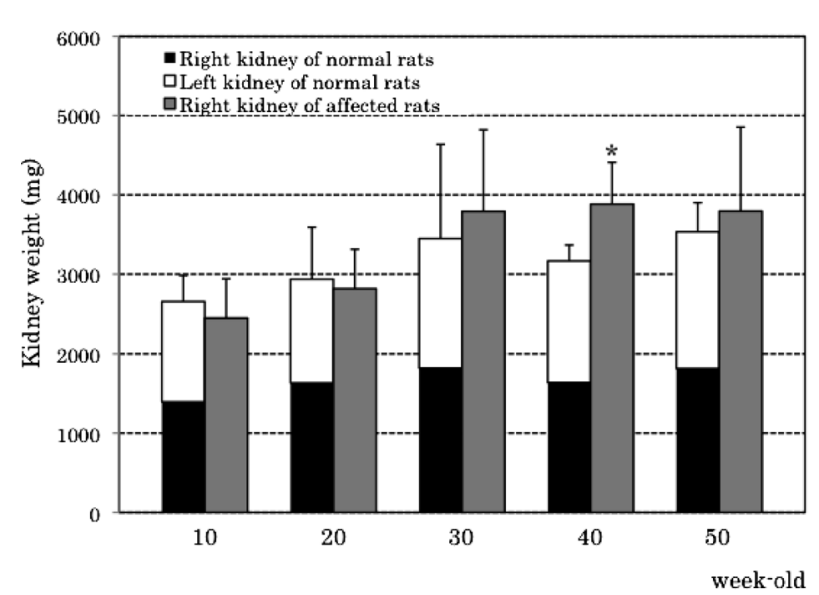

Fig. 1. Kidney weights in normal and affected rats. Data represent mean total renal weight (boxes) and SD (vertical bar). The right kidneys of affected rats (gray boxes) were significantly heavier than those of normal rats (black boxes) at all ages $(P<0.01)$. At 40 weeks, the weight of the remaining right kidneys in affected rats was significantly heavier than the total weight of right (black boxes) and left (white boxes) kidneys in normal rats $(* P<0.05)$.

By contrast, MCV (normal, $\mathrm{y}=-0.134 \mathrm{x}+60.94, \mathrm{R}^{2}=0.199$, $P=0.012 ;$ affected, $\mathrm{y}=-0.255 \mathrm{x}+67.27, \mathrm{R}^{2}=0.417$, $P<0.0001$ ), $\mathrm{MCH}$ (normal, $\mathrm{y}=-0.094 \mathrm{x}+21.33, \mathrm{R}^{2}=0.415$, $P<0.0001$; affected, $\mathrm{y}=-0.153 \mathrm{x}+24.18, \mathrm{R}^{2}=0.658$, $P<0.0001$ ), and MCHC (normal, $\mathrm{y}=-0.087 \mathrm{x}+35.12$, $\mathrm{R}^{2}=0.275, P=0.0025$; affected, $\mathrm{y}=0.113+36.19, \mathrm{R}^{2}=0.502$, $P<0.0001)$ gradually decreased with age in both normal and affected rats. The numbers of RBCs were significantly lower in affected than in normal rats at $10(P<0.01), 20$ $(P<0.001)$ and $30(P<0.05)$ weeks of age. In addition, the Hct of affected rats was significantly lower at $10(P<0.05)$ and $20(P<0.05)$ weeks, and the overall elevations of RBC and Hct were significantly lower in affected than in normal rats $(P<0.01)$. Normal and affected rats did not differ signif- icantly in $\mathrm{Hb}$ concentration, except at 30 weeks $(P<0.05)$. $\mathrm{MCV}$ at $10(P<0.05)$ and $20(P<0.01)$ weeks and $\mathrm{MCH}$ at 10 $(P<0.05), 20(P<0.001)$ and $40(P<0.05)$ weeks were significantly higher in affected than in normal rats, and the overall elevation of MCV $(P<0.01)$ and the regression slope (in absolute value) of $\mathrm{MCH}(P<0.05)$ were significantly greater in affected than in normal rats. The overall elevation and slope of MCHC were comparable in normal and affected rats.

Blood clinical chemistry (Table 3): Although plasma ALB level decreased with age in both normal $(\mathrm{y}=$ $\left.0.019 \mathrm{x}+3.38, \mathrm{R}^{2}=0.350, P=0.0006\right)$ and affected $(\mathrm{y}=-$ $\left.0.029 \mathrm{x}+3.26, \mathrm{R}^{2}=0.660, P<0.0001\right)$ rats, ALB concentrations were significantly $(P<0.01)$ lower in affected than in normal rats at 20,30, and 50 weeks. Plasma TCHO level increased with age in both normal $(y=1.882 x+49.37$, $\left.\mathrm{R}^{2}=0.500, P<0.0001\right)$ and affected $(\mathrm{y}=6.743 \mathrm{x}+12.96$, $\left.\mathrm{R}^{2}=0.627, P<0.0001\right)$ rats, though the slope of the increase was significantly $(P<0.0001)$ greater in affected than in normal rats, and the TCHO concentration was significantly $(P<0.001)$ higher in affected than in normal rats after 30 weeks of age. Although plasma Cre and UN concentrations in normal rats were unchanged with age (no significant correlation, Cre $\mathrm{R}^{2}=0.015, P=0.515$; $\mathrm{UN} \mathrm{R}^{2}=0.005, P=0.713$ ), both increased gradually with age in affected rats (Cre $\mathrm{y}=0.012 \mathrm{x}+0.643, \mathrm{R}^{2}=0.384, P<0.0001 ; \mathrm{UN} \mathrm{y}=0.385 \mathrm{x}+$ 18.94, $\left.\mathrm{R}^{2}=0.329, P=0.0004\right)$. Plasma Cre concentrations were significantly higher in affected than in normal rats at $40(P<0.01)$ and $50(P<0.001)$ weeks of age, and plasma UN concentrations were significantly higher in affected than in normal at 30, $40(P<0.01)$, and $50(P<0.05)$ weeks.

Plasma Ca concentrations gradually increased with age in normal $\left(\mathrm{y}=0.029 \mathrm{x}+8.863, \mathrm{R}^{2}=0.293, P=0.0020\right)$ and affected $\left(\mathrm{y}=0.044 \mathrm{x}+8.995, \mathrm{R}^{2}=0.386, P<0.0001\right)$ rats. Plasma $\mathrm{Ca}$ levels were significantly higher in affected than in normal rats at 30 and $40(P<0.01)$ weeks. There was no significant regression with age in plasma IP concentrations of normal $\left(\mathrm{R}^{2}=0.027, P=0.382\right)$ and affected $\left(\mathrm{R}^{2}=0.0008\right.$,

Table 2. Age-related alterations of hematological features in normal and affected male rats

\begin{tabular}{cllllll}
\hline Parameters & Phenotype & 10 weeks & 20 weeks & 30 weeks & 40 weeks & 50 weeks \\
\hline RBC & Normal & $6.37 \pm 0.44$ & $7.34 \pm 0.48$ & $7.52 \pm 0.67$ & $7.62 \pm 0.14$ & $7.94 \pm 0.74$ \\
$\left(10^{6} / \mu l\right)$ & Affected & $\left.5.64 \pm 0.38^{\mathrm{e}}\right)$ & $6.11 \pm 0.18^{\mathrm{f}}$ & $6.94 \pm 0.44^{\mathrm{d})}$ & $7.13 \pm 0.72$ & $7.14 \pm 0.42$ \\
\hline $\mathrm{Hct}$ & Normal & $40.0 \pm 2.9$ & $41.1 \pm 2.1$ & $41.8 \pm 3.5$ & $42.9 \pm 2.2$ & $43.4 \pm 2.9$ \\
$(\%)$ & Affected & $37.1 \pm 2.1^{\mathrm{d})}$ & $38.2 \pm 1.9^{\mathrm{d})}$ & $39.5 \pm 2.5$ & $41.4 \pm 5.2$ & $40.6 \pm 3.3$ \\
\hline $\mathrm{Hb}$ & Normal & $13.5 \pm 0.8$ & $13.6 \pm 1.3$ & $14.0 \pm 1.0$ & $13.2 \pm 0.7$ & $13.3 \pm 0.7$ \\
$(\mathrm{~g} / \mathrm{d} l)$ & Affected & $13.0 \pm 0.5$ & $13.0 \pm 0.3$ & $12.8 \pm 0.9^{\mathrm{d})}$ & $13.2 \pm 1.6$ & $12.4 \pm 0.8$ \\
\hline $\mathrm{MCV}$ & Normal & $62.8 \pm 0.9$ & $56.2 \pm 2.2$ & $55.9 \pm 4.1$ & $56.3 \pm 3.3$ & $54.9 \pm 3.4$ \\
$(\mathrm{f} l)$ & Affected & $\left.65.9 \pm 4.5^{\mathrm{a}}\right)$ & $62.6 \pm 3.0^{\mathrm{b})}$ & $57.0 \pm 3.2$ & $57.9 \pm 2.7$ & $57.0 \pm 4.0$ \\
\hline $\mathrm{MCH}$ & Normal & $21.2 \pm 1.4$ & $18.6 \pm 1.0$ & $18.8 \pm 2.1$ & $17.3 \pm 0.9$ & $16.8 \pm 0.9$ \\
$(p \mathrm{~g})$ & Affected & $23.2 \pm 1.7^{\mathrm{a})}$ & $21.2 \pm 0.7^{\mathrm{c})}$ & $18.5 \pm 1.2$ & $18.4 \pm 0.8^{\mathrm{a})}$ & $17.5 \pm 1.6$ \\
\hline $\mathrm{MCHC}$ & Normal & $33.8 \pm 2.2$ & $33.1 \pm 1.8$ & $33.6 \pm 2.2$ & $30.9 \pm 1.3$ & $30.7 \pm 0.9$ \\
$(\mathrm{~g} / \mathrm{d} l)$ & Affected & $35.2 \pm 1.9$ & $34.0 \pm 1.0$ & $32.5 \pm 1.2$ & $31.9 \pm 1.2$ & $30.7 \pm 1.8$ \\
\hline
\end{tabular}

Each data is represented as mean \pm SD. Significantly higher [a) $P<0.05$, b) $P<0.01$, and c) $P<0.001$ ] and significantly lower [d) $P<0.05$, e) $P<0.01$, and f) $P<0.001]$ than in aged matched normal rats by Student's $t$-tests. 
Table 3. Plasma concentrations of ALB, TCHO, Cre, UN, Ca, IP, and Mg in normal and affected rats

\begin{tabular}{ccccccc}
\hline Parameters & Phenotype & 10 weeks & 20 weeks & 30 weeks & 40 weeks & 50 weeks \\
\hline $\mathrm{ALB}$ & Normal & $3.1 \pm 0.1$ & $3.4 \pm 0.2$ & $2.6 \pm 0.2$ & $2.5 \pm 0.4$ & $2.5 \pm 0.3$ \\
$(\mathrm{~g} / \mathrm{d} l)$ & Affected & $3.0 \pm 0.2$ & $2.8 \pm 0.2^{\mathrm{e})}$ & $2.2 \pm 0.3^{\mathrm{e})}$ & $2.3 \pm 0.3$ & $2.0 \pm 0.1^{\mathrm{e})}$ \\
\hline $\mathrm{TCHO}$ & Normal & $70.8 \pm 5.5$ & $82.8 \pm 18.6$ & $107.1 \pm 29.1$ & $127.3 \pm 26.6$ & $142.0 \pm 31.3$ \\
$(\mathrm{mg} / \mathrm{d} l)$ & Affected & $77.2 \pm 7.9$ & $110.2 \pm 35.3$ & $245.3 \pm 104.7^{\mathrm{c})}$ & $286.2 \pm 45.3^{\mathrm{c})}$ & $317.1 \pm 14.3^{\mathrm{c})}$ \\
\hline $\mathrm{Cre}$ & Normal & $0.84 \pm 0.14$ & $0.89 \pm 0.14$ & $0.83 \pm 0.35$ & $0.93 \pm 0.11$ & $0.91 \pm 0.07$ \\
$(\mathrm{mg} / \mathrm{d} l)$ & Affected & $0.81 \pm 0.16$ & $0.82 \pm 0.08$ & $0.95 \pm 0.24$ & $\left.1.27 \pm 0.24^{\mathrm{b}}\right)$ & $\left.1.21 \pm 0.06^{\mathrm{c}}\right)$ \\
\hline $\mathrm{UN}$ & Normal & $20.0 \pm 3.1$ & $25.8 \pm 4.6$ & $22.5 \pm 2.5$ & $25.6 \pm 3.2$ & $20.6 \pm 1.3$ \\
$(\mathrm{mg} / \mathrm{d} l)$ & Affected & $21.7 \pm 1.7$ & $25.7 \pm 2.6$ & $31.8 \pm 9.8^{\mathrm{b})}$ & $38.8 \pm 6.5^{\mathrm{b})}$ & $32.4 \pm 6.8^{\mathrm{a})}$ \\
\hline $\mathrm{Ca}$ & Normal & $9.4 \pm 0.2$ & $9.5 \pm 0.7$ & $9.4 \pm 0.5$ & $10.0 \pm 0.6$ & $10.4 \pm 0.6$ \\
$(\mathrm{mg} / \mathrm{d} l)$ & Affected & $9.6 \pm 0.4$ & $9.2 \pm 0.7$ & $10.3 \pm 0.4^{\mathrm{b})}$ & $11.5 \pm 1.0^{\mathrm{b})}$ & $10.7 \pm 0.5$ \\
\hline $\mathrm{IP}$ & Normal & $9.5 \pm 0.9$ & $9.6 \pm 2.7$ & $7.6 \pm 0.9$ & $9.7 \pm 2.4$ & $8.2 \pm 1.1$ \\
$(\mathrm{mg} / \mathrm{d} l)$ & Affected & $9.7 \pm 1.3$ & $8.4 \pm 1.4$ & $9.3 \pm 2.4^{\mathrm{b})}$ & $10.7 \pm 2.5$ & $8.4 \pm 2.8$ \\
\hline $\mathrm{Mg}$ & Normal & $2.1 \pm 0.3$ & $2.9 \pm 0.4$ & $2.1 \pm 0.3$ & $3.0 \pm 0.7$ & $2.8 \pm 0.6$ \\
$(\mathrm{mg} / \mathrm{d} l)$ & Affected & $2.2 \pm 0.3$ & $2.3 \pm 0.3^{\mathrm{d})}$ & $3.1 \pm 0.5^{\mathrm{c})}$ & $4.3 \pm 0.8^{\mathrm{a})}$ & $3.0 \pm 0.4$ \\
\hline
\end{tabular}

Each data is represented as mean \pm SD. Significantly higher [a) $P<0.05$, b) $P<0.01$, and c) $P<0.001$ ] and significantly lower [e) $P<0.01]$ than in aged matched normal rats by Student's $t$-tests.

Table 4. Age-related alterations in Cre clearance, fractional UN excretion (FEUN), and daily urinary protein excretion in normal and affected male rats

\begin{tabular}{ccccccc}
\hline Parameters & UUA & 10 weeks & 20 weeks & 30 weeks & 40 weeks & 50 weeks \\
\hline $\begin{array}{c}\text { Cre clearance } \\
(\mathrm{m} / \mathrm{min} / \mathrm{kg})\end{array}$ & Normal & $3.85 \pm 1.30$ & $4.77 \pm 0.91$ & $3.50 \pm 1.75$ & $2.14 \pm 0.54$ & $2.53 \pm 0.44$ \\
\hline AEfected & $4.73 \pm 1.50$ & $4.25 \pm 0.71$ & $3.02 \pm 1.02$ & $2.15 \pm 0.60$ & $2.25 \pm 0.32$ \\
$(\%)$ & Normal & $125.7 \pm 42.1$ & $76.3 \pm 12.0$ & $81.0 \pm 26.9$ & $83.8 \pm 17.0$ & $102.2 \pm 18.9$ \\
\hline Protein excretion & Norfected & $64.0 \pm 21.4^{\mathrm{c})}$ & $53.1 \pm 6.1^{\mathrm{c})}$ & $57.8 \pm 20.2^{\mathrm{b})}$ & $63.2 \pm 9.0^{\mathrm{b})}$ & $83.3 \pm 15.9$ \\
$(\mathrm{mg} / \mathrm{kg} /$ day $)$ & Affected & $126.6 \pm 20.4$ & $265.6 \pm 264.0$ & $237.3 \pm 255.4$ & $319.4 \pm 251.8$ & $217.0 \pm 99.6$ \\
\hline
\end{tabular}

Each data is represented as mean \pm SD. Significantly higher [a) $P<0.001]$ and significantly lower [b) $P<0.05$ and c) $P<0.01]$ than in aged matched normal rats by Student's $t$-tests.

$P=0.872)$ rats, though plasma IP concentrations were significantly higher $(P<0.05)$ in affected than in normal rats at 30 weeks. Although plasma $\mathrm{Mg}$ concentration remained almost constant with age in normal rats $\left(\mathrm{R}^{2}=0.073\right.$, $P<0.150)$, it gradually increased with age in affected rats $\left(\mathrm{y}=0.039+1.831, \mathrm{R}^{2}=0.369, P=0.0001\right)$ and was significantly higher in affected rats than in normal rats at 30 $(P<0.001)$ and $40(P<0.05)$ weeks.

Cre clearance and fractional excretion of UN (Table 4): Cre clearance gradually decreased with age in both normal $\left(\mathrm{y}=-0.057 \mathrm{x}+5.115, \mathrm{R}^{2}=0.266, P=0.003\right)$ and affected $\left(\mathrm{y}=0.073 \mathrm{x}+5.418, \mathrm{R}^{2}=0.448, P<0.0001\right)$ rats, and the Cre clearance of affected rats was comparable to that of normal rats at all ages. The fractional excretion of UN $(\%, 100 \times$ $\mathrm{UN}$ clearance/Cre clearance) was lower in affected rats than in normal rats at all ages and these differences were significant at 10-40 weeks $(P<0.05)$. These results indicate that, $\mathrm{UN}$ excretion was more impaired than Cre excretion in affected rats.

Urinary protein analyses (Table 4 and Fig. 2): Daily uri- nary protein excretion increased with age in affected ( $\left.\mathrm{y}=29.4 \mathrm{x}-1.687, \mathrm{R}^{2}=0.522, P<0.0001\right)$ but not in normal $\left(\mathrm{R}^{2}=0.010, P=0.590\right)$ rats, and was significantly (3-5-fold, $P<0.001)$ greater in affected than in normal rats after 30 weeks of age (Table 4). Polyacrylamide gel electrophoresis of urinary proteins of 10 week-old rats revealed a $66 \mathrm{kDa}$ band, which was likely albumin [29, 30]. Although the number and intensity of the signals varied among individual animals, the albumin band was more intense in the urine of 10-50 week-old affected than of age-matched normal rats (Fig. 2).

Renal histology: Pathological alterations observed in the remaining right kidney of affected rats aged 10 to 30 weeks were similar to those previously reported [2]. A PAS-positive substance started to accumulate in a few glomeruli of 10 week-old animals, and glomerular hypertrophy, focal segmental or global glomerulosclerosis, and dilated tubules containing cast formation were observed in renal cortices at 30 weeks (data not shown). At 50 weeks of age, some segmental glomerulosclerosis and cast formation were detected 


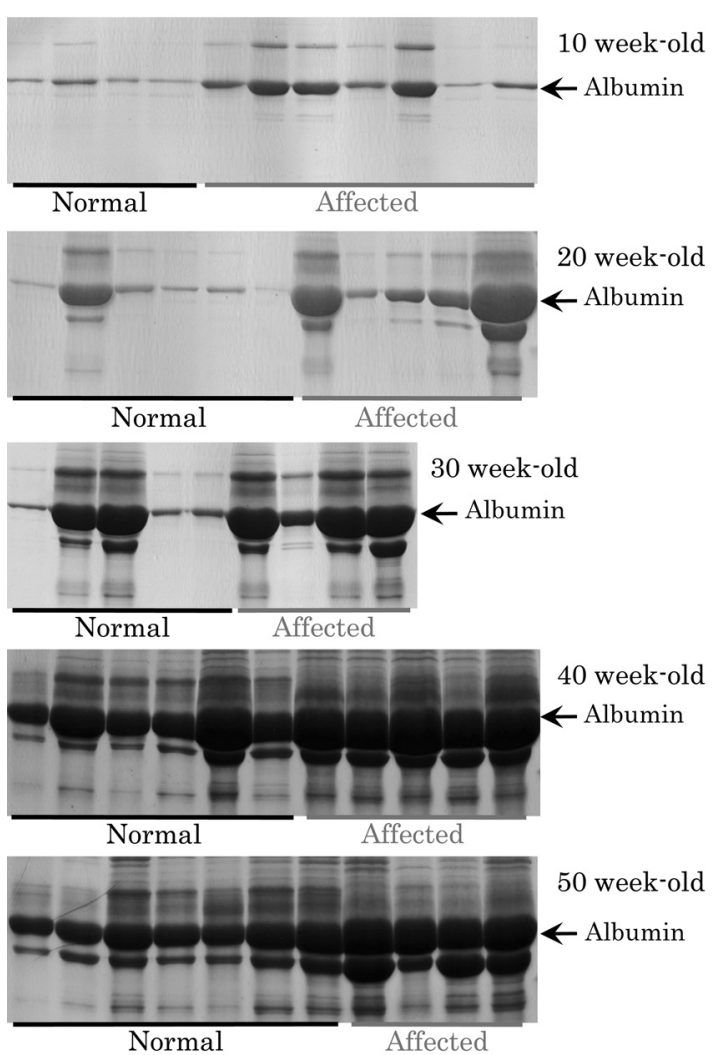

Fig. 2. SDS-PAGE of urinary proteins. Five microliters of urine from each rat was applied to each lane. The major signal corresponding to albumin $(66 \mathrm{kDa})$ was detected in the urine of 10 week-old rats. Signals gradually increased with age in both normal and affected rats. Although the intensity of signals varied considerably among the samples, overt albuminuria was more often detected in affected than in normal rats. Although intense albumin signals were detected in all 40 and 50 week-old rats, the intensities of the signals were higher in affected than in normal rats.

in the kidneys, even of normal rats (Fig. 3A-D), whereas the right kidneys of affected rats showed more severe types of glomerular sclerosis, glomerular crescent, and cast formation, as well as severe interstitial fibrosis in the renal medulla (Fig. 4A-F). These findings show that, in the remaining kidneys of affected rats, pathological alterations deteriorated with age and extended to the medullary region at advanced age.

Number of glomeruli in right kidneys (Table 5): The mean weight of the right kidneys of affected rats used to determine number of glomeruli was approximately 1.7-fold greater than that of normal rats, and this difference was statistically significant $(P<0.05)$. The number of glomeruli in the right kidneys was approximately 1.2 -fold greater in affected than in normal rats, and this difference was also statistically significant $(P<0.01)$. In affected rats, the weight associated with individual nephrons (kidney weight divided by glomerular number) increased.

\section{DISCUSSION}

Since the affected rats of the UUA strain had normal body weight and normal appearance at all age examined, the congenital loss of one kidney was not considered a problem for maintaining general body condition and growth. In this study, however, we found that these affected rats had already shown signs of polydipsia and polyuria at 10 weeks of age, with these abnormalities deteriorating over time. Polyuria has been reported not only in uninephrectomized rats [15] but also in animal models exhibiting bilateral hypoplasia of the renal parenchyma $[30,37]$. Therefore, the failure to concentrate urine may be a common feature associated with congenitally reduced nephron mass. A decrease in nephron number has been shown to cause hyperfiltration in the remaining glomeruli [31]. Long-term urinary overload of the tubules resulting from hyperfiltration may exceed the concentration capacity of these nephrons.

The decreased renal production of erythropoietin (EPO) is a primary cause of anemia in humans [17] and experimental animals [35] with renal failure. As 40-50 week-old affected UUA rats had normal values of RBC and Hct, the presence of erythropenia in 10-30 week-old animals was unexpected. In contrast to normocytic and normochromic anemia in glomerulonephritis (ICGR) mice [36], blood $\mathrm{Hb}$ concentration in affected UUA rats was maintained at an almost normal level, and it was accompanied by macrocytic hyperchromic erythropenia. These results suggest that the decrease in oxygen transport resulting from erythropenia may be partially compensated by an increase in $\mathrm{Hb}$ contents in individual RBCs of large volume. Increased RBC volume and $\mathrm{Hb}$ content may be secondary to a reduced proliferation of erythrocytic progenitor cells due to relatively low level of EPO or possible inhibitors of erythropoiesis [8].

The co-occurrence of hypoalbuminemia and hypercholesterolemia has been also reported in human patients with nephrotic syndrome [3], ICGR mice [20], and puromycin aminonucleoside-induced nephrotic rats [32]. The severity of hypercholesterolemia tends to correlate with the severity of hypoalbuminemia in nephritic syndrome [3]. We found that plasma TCHO was negatively correlated with plasma ALB in affected UUA rats (data not shown). In general, renal hyperparathyroidism is the result of decreased excretion of phosphorus and a defect in vitamin $\mathrm{D}_{3}$ hydroxylation in patients with chronic renal failure. As neither hyperphosphatemia nor hypocalcemia was obvious in affected UUA rats, these rats may not have a severe defect in vitamin $D_{3}$ hydroxylation and any renal hyperparathyroidism present may be mild. In patients with chronic renal failure, hypermagnesemia is associated with a considerable reduction in GFR [18]. Our finding, that age-related changes in plasma $\mathrm{Mg}$ concentration paralleled the changes in plasma Cre in affected UUA rats, suggests that the elevated Mg concentrations is probably caused by a decrease in renal excretive function. 

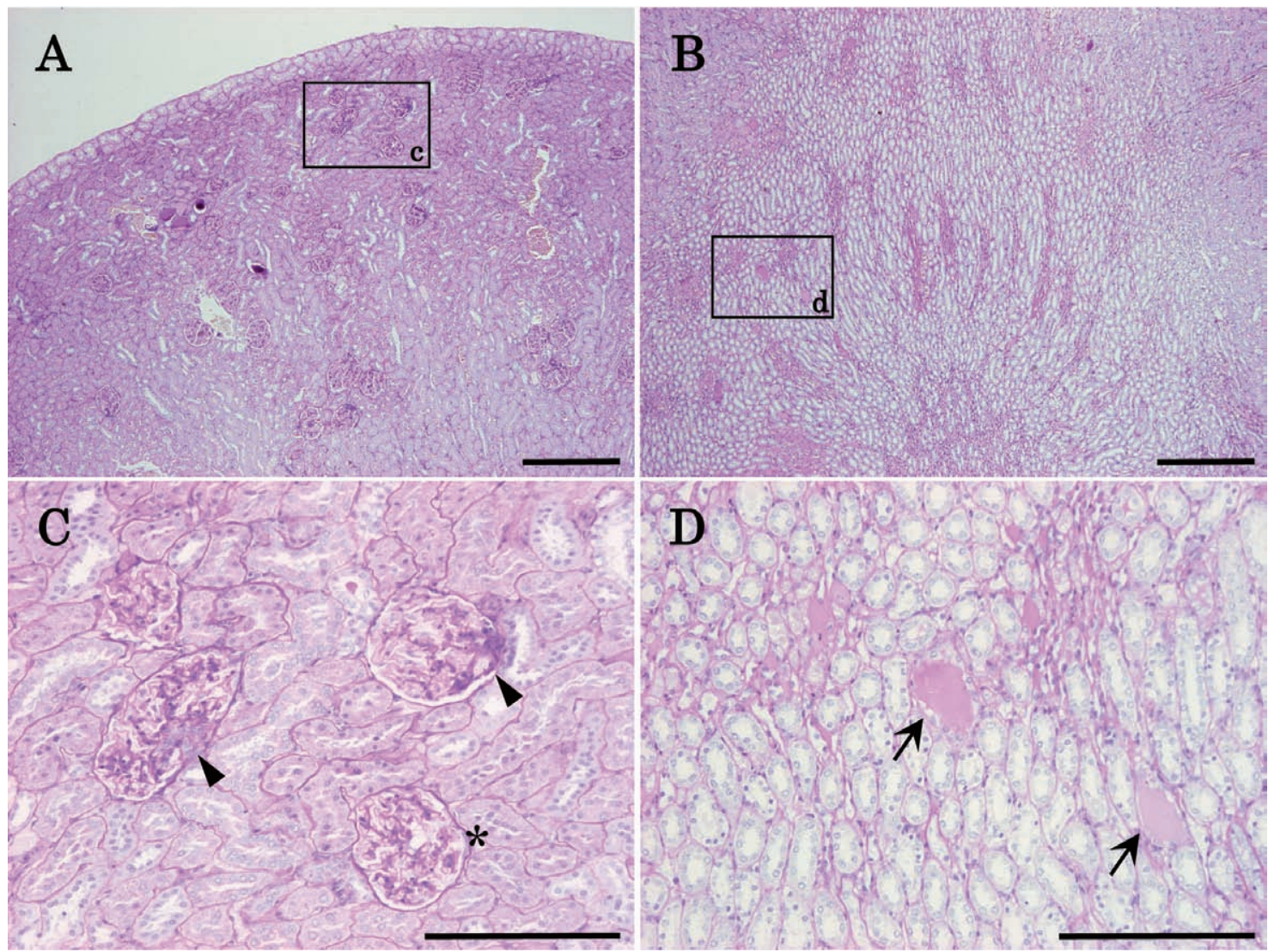

Fig. 3. Microscopic findings of the right kidney in normal (A-D) at 50 week of age. A, Low magnification of renal cortex. B, Low magnification of renal medulla. C, High magnification of square $\mathrm{c}$ in A. Normal (asterisk) and partially sclerotic glomeruli (solid arrowheads) are shown. D, High magnification of square $\mathrm{d}$ in B. Cast formation was present in a few collecting ducts. PAS staining. Scale bar, 500 (A, B) and 200 (C, D) $\mu \mathrm{m}$.

Cre clearance gradually decreased with age in affected UUA rats and was comparable to that of normal at all ages, despite the age-related increase and significantly higher plasma Cre concentrations in 40 and 50 week-old affected than normal rats. Urinary Cre excretion was maintained at a steady state level after 30 weeks in affected rats, but decreased with age in normal rats. Thus, the degree of GFR reduction in 10-30 week-old affected rats might be too small to detect by Cre clearance analysis, and the tubular Cre secretion might increase with increase of plasma Cre concentration in 40-50 week-old affected animals [7]. In contrast, the fractional excretion of UN was significantly lower in affected than in normal rats at almost all ages examined. In general, fractional excretion of UN decreases when urea permeability is increased [25]. Water loss resulting from glomerular hyperfiltration in the individual nephrons may increase vasopressin secretion and urea permeability in the collecting ducts of affected rats.

SDS-PAGE showed that albumin was the major urinary protein. Although molecular size of albumin allows it to pass through the glomerular filtration barrier, its leakage is small, due to its negative charge, and small amounts of albumin are detected in the urine from normal adult rats [19].
Since the urine of affected UUA rats is diluted compared with the urine of normal rats, the amount of albumin leaking into the urine was much greater in affected than in normal rats. In addition, leakage of proteins of higher molecular weight than albumin was greater in affected than in normal rats, suggesting that the glomerular filtration barrier was impaired in affected rats, and that this damage increased with age.

Uninephrectomy of young rats has been reported to cause podocyte damages, which are thought to result from the maladaptation of podocytes to the increasing glomerular volume and to be a trigger of glomerular sclerosis [16]. Congenital glomerular hyperfiltration in the remaining kidney of affected rats may also provide long-term mechanical stress on the glomerular slit membrane consisting of a network of podocytes and basement membrane. As we observed age-related glomerular lesions in the right kidneys of 50 week-old normal rats, the remarkable alterations in the remaining kidneys of older affected rats were not only sever glomerular damages in the renal cortices but also medullar lesions. Severe proteinuria has been reported to cause a tubular disorder, considered a major factor for progressive chronic renal failure [1]. Thus, in affected UUA rats, severe 

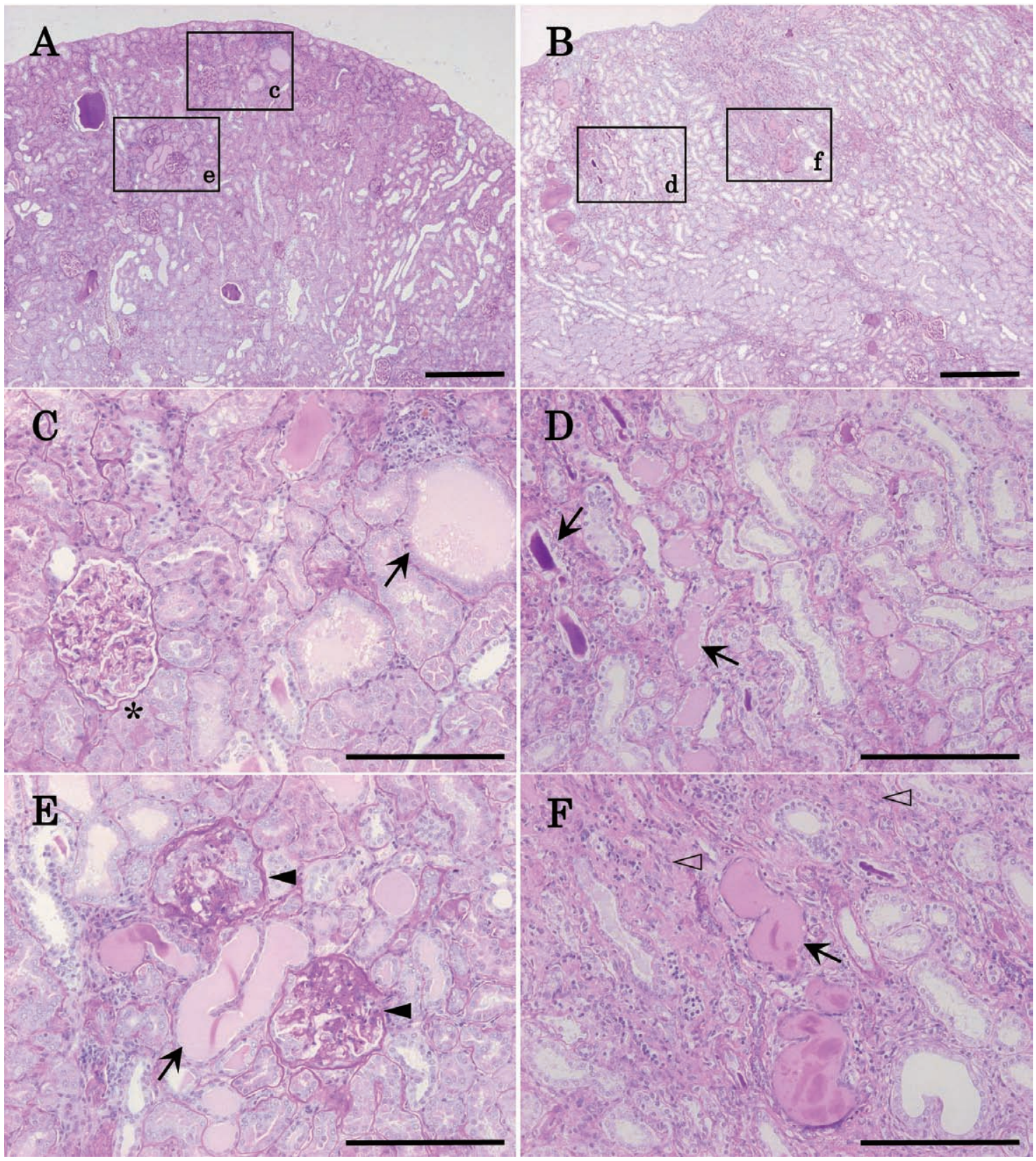

Fig. 4. Microscopic findings of the right kidney in affected (A-F) rats at 50 week of age. A, Low magnification of renal cortex. B, Low magnification of renal medulla. C, High magnification of square $\mathrm{c}$ in A. Some glomeruli were considered normal (asterisk) and cast formation occurred in many tubules (arrow). D, High magnification of square d in B. Hyaline casts were observed in some tubules (arrows). E, High magnification of square e in A, showing global sclerosis and glomerular crescent (solid arrowhead). Cast formations were present in many tubules (arrow). F, High magnification of square $\mathrm{f}$ in $\mathrm{B}$. In addition to cast formation (arrow), severe interstitial fibrosis was observed (open arrowheads). PAS staining. Scale bar, 500 (A, B) and $200(\mathrm{C}, \mathrm{D}, \mathrm{E}, \mathrm{F}) \mu \mathrm{m}$.

proteinuria due to glomerular sclerosis may stress the tubular epithelium and induce interstitial infiltration, which may impair tubular concentration capacity.

Enlargement of the remaining kidney has been observed in human fetuses $[9,10,14]$ and patients [13] with a congenitally or functionally solitary kidney. In addition to being heavier than both kidneys of normal rats, the remaining right kidney in affected rats had significantly increased numbers of nephrons. Since the number of glomeruli in the remaining kidney of affected rats was not equal to that in both kidneys of normal rats, the individual nephrons in affected rats were heavier, indicating that individual nephrons in affected 
Table 5. Wet weight and number of glomeruli in the right kidneys of normal and affected adult males

\begin{tabular}{lcc}
\hline & Normal & Affected \\
\hline Kidney weight $(\mathrm{KW})(\mathrm{mg})$ & $1466.9 \pm 199.7$ & $\left.2454.9 \pm 517.6^{\mathrm{a}}\right)$ \\
Glomerular number $(\mathrm{GN})$ & $20611.4 \pm 5803.3$ & $25888.0 \pm 2188.2^{\mathrm{b})}$ \\
$\mathrm{KW} / \mathrm{GN}^{*}(\mu \mathrm{g})$ & $75.5 \pm 21.2$ & $94.6 \pm 16.9$ \\
\hline
\end{tabular}

*: Weight associated with individual nephrons. Each data is represented as mean \pm SD. Significantly higher [a) $P<0.05$, b) $P<0.01$ ] than in normal rats by Student's $t$-tests.

rats were hypertrophic. Although $\mathrm{UNx}$ in rats during the early postnatal period failed to induce additional nephrogenesis in the remaining kidney $[11,12]$, prenatal UNx in sheep resulted in an increased number of nephrons in the remaining kidney [6]. In contrast to postnatal UNx, affected UUA rats congenitally lack their left kidneys. Thus, an increased number of nephrons during the embryonic and/or postnatal nephrogenic period may contribute to CRG in the remaining kidney. In future investigations, we will assess the timing of nephron endowment in the remaining kidneys of UUA rats.

ACKNOWLEDGMENT. This work was supported in part by a Grant-in-Aid for Scientific Research to H. Suzuki (No. 19580350 and No. 22580341) from the Ministry of Education, Culture, Sports, Science and Technology of Japan.

\section{REFERENCES}

1. Abbate, M., Zoja, C. and Remuzzi, G. 2006. How does proteinuria cause progressive renal damage? J. Am. Soc. Nephrol. 17: 2974-2984.

2. Amakasu, K., Suzuki, K. and Suzuki, H. 2009. The unilateral urogenital anomalies (UUA) rat: a new mutant strain associated with unilateral renal agenesis, cryptorchidism, and malformations of reproductive organs restricted to the left side. Comp. Med. 59: 249-256.

3. Appel, G. B., Blum, C. B., Chien, S., Kunis, C. L. and Appel, A. S. 1985. The hyperlipidemia of the nephritic syndrome. Relation to plasma albumin concentration, oncotic pressure and viscosity. N. Eng. J. Med. 312: 1544-1548.

4. Argueso, L. R., Ritchey, M. L., Boyle, E. T. Jr., Milliner, D. S., Bergstralh, E. J. and Kramer, S. A. 1992. Prognosis of patients with unilateral renal agenesis. Pediatr. Nephrol. 6: 412-416.

5. Celsi, G., Bohman, S. O. and Aperia, A. 1987. Development of focal glomerulosclerosis after unilateral nephrectomy in infant rats. Pediatr. Nephrol. 1: 290-296.

6. Douglas-Denton, R., Moritz, K. M., Bertram, J. F. and Wintour, E. M. 2002. Compensatory renal growth after unilateral nephrectomy in the ovine fetus. J. Am. Soc. Nephrol. 13: 406410.

7. Eisner, C., Faulhaber-Walter, R., Wang, Y., Leeiahavanichkul, A., Yuen, P. S., Mizel, D., Star, R. A., Briggs, J. P., Levine, M. and Schnermann, J. 2010. Major contribution of tubular secretion to creatnine clearance in mice. Kidney Int. 77: 519-526.

8. Fukushima, Y., Fukuda, M., Yoshida, K., Yamaguchi, A., Nakamoto, Y., Miura, A. B., Harada, T. and Tsuchida, S. 1986. Serum Erythropoietin levels and inhibitors of erythropoiesis in patients with chronic renal failure. Tohoku J Exp Med. 150: 115.

9. Glazebrook, K. N., McGrath, F. P. and Steele, B. T. 1993. Prenatal compensatory renal growth: documentation with US. Radiology 189: 733-735.

10. Hill, L. M., Nowak, A., Hartle, R. and Tush, B. 2000. Fetal compensatory renal hypertrophy with a unilateral functioning kidney. Ultrasound Obstet. Gynecol. 15: 191-193.

11. Kaufman, J. M., Hardy, R. and Hayslett, J. P. 1975. Agedependent characteristics of compensatory renal growth. Kidney Int. 8: 21-26.

12. Larsson, L., Aperia, A. and Wilton, P. 1980. Effect of normal development on compensatory renal growth. Kidney Int. 18: 29-35.

13. Maluf, N. S. 1997. On the enlargement of the normal congenitally solitary kidney. Br. J. Urol. 79: 836-841.

14. Mandell, J., Peters, C. A., Estroff, J. A., Allred, E. N. and Benacerraf, B. R. 1993. Human fetal compensatory renal growth. $J$. Urol. 150: 790-792.

15. Mulroney, S. E., Woda, C., Johnson, M. and Pesce, C. 1999. Gender differences in renal growth and function after uninephrectomy in adult rats. Kidney Int. 56: 944-953.

16. Nagata, M. and Kriz, W. 1992. Glomerular damage after uninephrectomy in young rats. II. Mechanical stress on podocytes as a pathway to sclerosis. Kidney Int. 42: 148-160.

17. Nangaku, M. and Eckardt, K. U. 2006. Pathogenesis of renal anemia. Semin. Nephrol. 26: 261-268.

18. Navarro-González, J. F., Mora-Fernández, C. and GarcíaPérez, J. 2009. Clinical implications of disordered magnesium homeostasis in chronic renal failure and dialysis. Semin. Dial. 22: $37-44$

19. Neuhaus, O. W. and Flory, W. 1978. Age-dependent changes in the excretion of urinary proteins by the rat. Nephron 22: 570-576.

20. Ogura, A., Asano, T., Suzuki, O., Yamamoto, Y., Noguchi, Y., Kawaguchi, H. and Yamaguchi, Y. 1994. Hereditary nephritic syndrome with progression to renal failure in a mouse model (ICGN strain): clinical study. Nephron 68: 239-244.

21. Okuda, S., Motomura, K., Sanai, T., Tsuruda, H., Oh, Y., Onoyama, K. and Fujishima, M. 1987. Influence of age on deterioration of the remnant kidney in uninephrectomized rats. Clin. Sci. (Lond.) 72: 571-576.

22. Ormrod, D. and Miller, T. 1980. Experimental uremia. Description of a model producing varying degrees of stable uremia. Nephron 26: 249-254.

23. Phillipou, G., James, S. K., Seaborn, C. J. and Phillips, P. J. 1989. Screening for microalbuminuria by use of a rapid lowcost colorimetric assay. Clin. Chem. 35: 456-458.

24. Robson, W. L., Leung, A. K. and Rogers, R. C. 1995. Unilateral renal agenesis. Adv. Pediatr. 42: 575-592.

25. Silkensen, J. R. and Kasiske, B. L. 2004. Laboratory assessment of kidney disease: clearance, urinalysis, and kidney biopsy. pp. 1107-1150. In: The Kidney (Brenner, B. M. ed.), Philadelphia, Saunders.

26. Suzuki, H. and Suzuki, K. 1995. Pathophysiology and postnatal pathogenesis of hypoplastic kidney $(h p k / h p k)$ in the male hypogonadic mutant rat (hgn/hgn). J. Vet. Med. Sci. 57: 891897.

27. Suzuki, H. and Suzuki, K. 1998. Rat hypoplastic kidney (hpk/ hpk) induces renal anemia, hyperparathyroidism, and osteodystrophy at the end stage of renal failure. J. Vet. Med. Sci. 60: 1051-1058.

28. Suzuki, H., Takenaka, M. and Suzuki, K. 2007. Phenotypic 
characterization of spontaneously mutated rats showing lethal dwarfism and epilepsy. Comp. Med. 57: 360-369.

29. Suzuki, H., Tokuriki, T., Kamita, H., Oota, C., Takasu, M., Saito, K. and Suzuki, K. 2006. Age-related pathophysiological changes in rat oligomeganephronic hypoplastic kidney. Pediatr. Nephrol. 21: 637-642.

30. Suzuki, H., Tokuriki, T., Saito, K., Hishida, A. and Suzuki, K. 2005. Glomerular hyperfiltration and hypertrophy in the rat hypoplastic kidney as a model of oligomeganephronic disease. Nephrol. Dial. Transplant. 20: 1362-1369.

31. Taal, M. W., Luyckx, V. A. and Brenner, B. M. 2004. Adaptation to nephron loss. pp. 1955-1997. In: The Kidney (Brenner, B. M. ed.), Philadelphia, Saunders.

32. Thabet, M. A., Challa, A., Chan, J. C., Pandak, W. M., Heuman, D. M. and Vlahčevič, Z. R. 1993. Studies of alteration of hepatic cholesterol metabolism in puromycin-induced nephritic syndrome in rats. Kidney Int. 44: 789-794.

33. Woolf, A. S. and Hillman, K. A. 2007. Unilateral renal agene- sis and the congenital solitary functioning kidney: developmental, genetic, and clinical perspectives. BJU Int. 99: 17-21.

34. Woods, L. L., Weeks, D. A. and Rasch, R. 2001. Hypertension after neonatal uninephrectomy in rats precedes glomerular damage. Hypertension 38: 337-342.

35. Yamaguchi-Yamada, M., Manabe, N., Kiso, M., Goto, Y., Mori, T., Sakata, C., Anan, S., Nagao, M., Yamamoto, Y. and Ogura, A. 2005. Dysfunction of erythropoietin-producing interstitial cells in the kidneys of ICR-derived glomerulonephritis (ICGN) mice. J. Vet. Med. Sci. 67: 891-899.

36. Yamaguchi-Yamada, M., Manabe, N., Uchio-Yamada, K., Akashi, N., Goto, Y., Miyamoto, Y., Nagao, M., Yamamoto, Y., Ogura, A. and Miyamoto, H. 2004. Anemia with chronic renal disorder and disrupted metabolism of erythropoietin in ICR-derived glomerulonephritis (ICGN) mice. J. Vet. Med. Sci. 66: 423-431.

37. Zalups, R. K. 1993. The Os/+ mouse: a genetic animal model of reduced renal mass. Am. J. Physiol. 264: 53-60. 